\title{
An amplicon-based approach for sequencing of SARS-CoV-2 using COVIDSeq assay on Illumina MiSeq sequencing platforms
}

Rahul C. Bhoyar ${ }^{1}$, Vigneshwar Senthivel ${ }^{1,2}$, Bani Jolly ${ }^{1,2}$, Mohamed Imran $^{1,2}$, Abhinav Jain $^{1,2}$, Mohit Kumar Divakar ${ }^{1,2}$, Vinod Scaria ${ }^{1,2,3, *}$, Sridhar Sivasubbu $u^{1,2,3,4, * *}$

${ }^{1}$ CSIR- Institute of Genomics and Integrative Biology (CSIR-IGIB), Mathura Road, Delhi, 110025, India

${ }^{2}$ Academy of Scientific and Innovative Research (AcSIR), Ghaziabad, Uttar Pradesh, 201002, India

${ }^{3}$ Technical Contact

${ }^{4}$ Lead Contact

**Correspondence: sridhar@igib.in

*Correspondence: vinods@igib.in

\section{Summary}

Sequencing genomes of SARS-CoV-2 is crucial for understanding the genetic epidemiology of COVID19 pandemic. It is also critical for understanding the evolution of the virus and also for the rapid development of the diagnostic tools. The present protocol is the modification of the Illumina COVIDSeq amplicon based sequencing approach adapted for benchtop sequencers like MiSeq, iSeq and MiniSeq.

For complete details on the use and execution of this protocol, please refer to Bhovar et al. (2021). 


\section{Graphical Abstract}

SARS-CoV-2 surveillence with COVIDSeq approach using Illumina benchtop sequencers

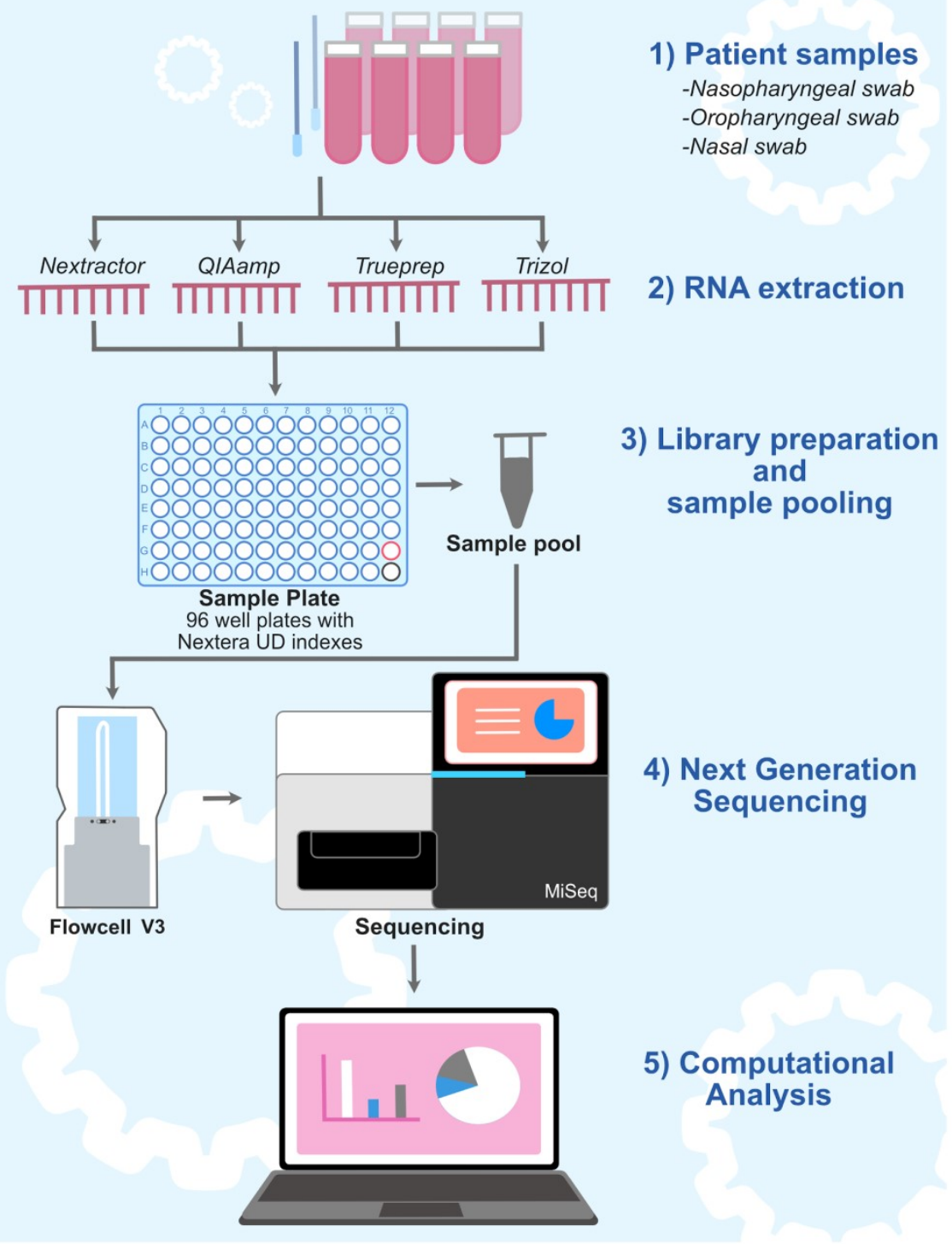




\section{Introduction}

The Illumina COVIDSeq assay is an amplicon-based next generation sequencing (NGS) approach for detection of the SARS-CoV-2 RNA isolated from nasopharyngeal, oropharyngeal, and mid-turbinate nasal swabs from patients. The assay is one of the first NGS based SARS-CoV-2 detection assays approved for use under U.S Food and Drug Administration's Emergency Use Authorization (EUA). The COVIDSeq test was first standardized and validated for Illumina NovaSeq sequencer and can accommodate 384 to 3072 samples depending on the configuration of flow cells and the instrument (Bhoyar et al. 2021). The protocol is compatible with all commonly used viral RNA isolation methods including QIAmp (QIAGEN), Nextractor ${ }^{\circledR}$ NX-48S (GENOLUTION), Trueprep (Molbio Diagnostics Pvt. Ltd.) and TRIzol (Invitrogen).

While originally adapted and optimised for larger sequencers, this assay could benefit from optimisation for smaller throughput benchtop equipments for smaller batch sizes. This manuscript details the protocol with marginal modifications and along with an open-source pipeline for the analysis and interpretation of data. The protocol is standardized for the Illumina MiSeq sequencing platform, however by considering the final loading concentration of the library and the data output, the proposed protocol can be adopted for any benchtop sequencer from Illumina Inc.

\section{Library Preparation Setup}

- Isolated SARS-CoV-2 RNA samples.

- A pre-amplification environment with a Biosafety level 2 (BSL-2) facility is required during RNA sample handling and cDNA preparation.

- Personal Protective Equipments (PPEs), as recommended by WHO for handling viral samples contagious to humans.

- Two 96-well PCR thermal cyclers.

- Thermo mixer with a 96-well PCR plate holder.

- Qubit HS dsDNA quantification kit along with instrument (Invitrogen).

\section{cDNA preparation}

\section{Timing: [1 hour]}

(All steps to be carried out within the Biosafety cabinet of the BSL-2 facility)

1. Annealing of RNA

a. Label a new 96 well PCR plate as CDNA plate (Fig.1).

b. Add $8.5 \mu \mathrm{l}$ of the isolated SARS-CoV-2 sample into the wells of the cDNA plate.

c. Add $8.5 \mu \mathrm{l}$ of EPH3 HT to each well.

d. Mix well with a P10 or P20 multichannel pipette.

e. Seal and spin the plate at $1000 \times \mathrm{g}$ for 1 minute.

f. Place the cDNA plate on a thermal cycler and run the following program

- Choose the preheat lid option

- Set the final volume of the reaction to be $17 \mu \mathrm{l}$

- $65^{\circ} \mathrm{C}$ for 3 minutes

- Hold at $4^{\circ} \mathrm{C}$ 


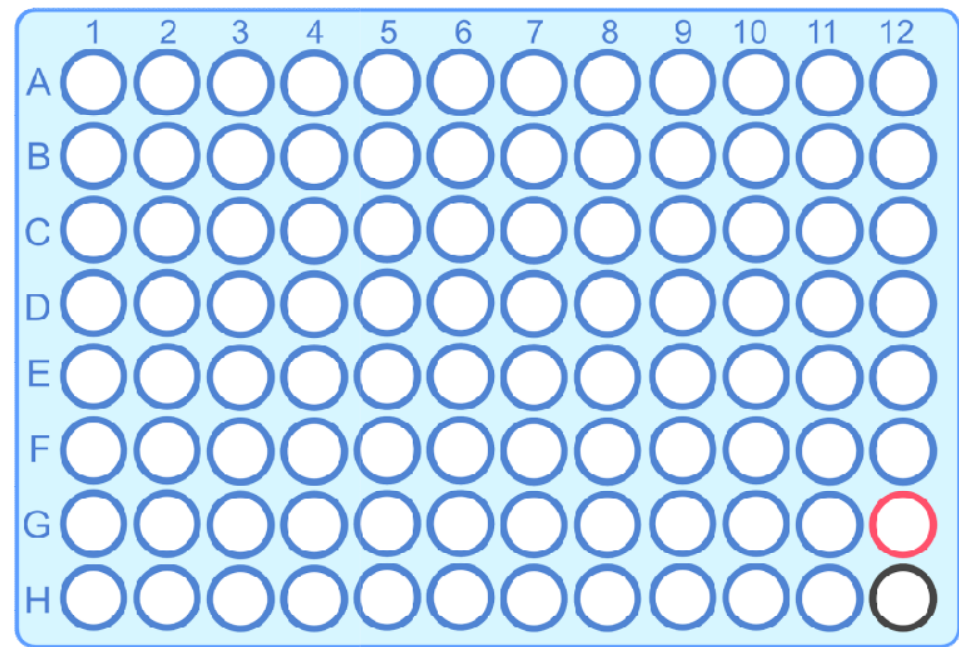

Sample Plate
Legend

Samples

Positive Control

Negative Control

Fig. 1: Layout of the sample plate to be used denoting the samples, PC and NC, up-to 94 samples can be processed at once. A positive control (CPC HT) and Negative Control is included in every plate that is processed using COVIDSeq protocol.

2. First strand cDNA synthesis

a. Prepare First strand cDNA Mastermix (for 96 samples) by mixing $864 \mu \mathrm{l}$ of FSM HT and $96 \mu \mathrm{l}$ of RVT HT in a $1.5 \mathrm{ml}$ microcentrifuge tube.

b. Add $8 \mu$ l of the First strand cDNA mastermix into each well of the cDNA plate and pipette well to mix.

c. Seal and spin the plate at $1000 \times \mathrm{g}$ for 1 minute.

d. Place the cDNA plate on a thermal cycler and run the following program

- Choose the preheat lid option

- Set the final volume of the reaction to be $25 \mu \mathrm{l}$

- $25^{\circ} \mathrm{C}$ for 5 minutes

- $50^{\circ} \mathrm{C}$ for 10 minutes

- $\quad 80^{\circ} \mathrm{C}$ for 5 minutes

- Hold at $4^{\circ} \mathrm{C}$

Pause Point: The procedure can be paused here and the cDNA plate can be stored at $-20^{\circ} \mathrm{C}$ for upto 7 days.

\section{Amplification of cDNA}

\section{Timing: [4 hours]}

3. Amplification of prepared cDNA

a. Label two 96-well PCR plates as COV1 and COV2.

b. Prepare PCR1 Mastermix and PCR2 Mastermix in two separate $15 \mathrm{ml}$ tubes.

- Add $1440 \mu \mathrm{l}$ of IPM HT (15 $\mu \mathrm{l} \times 96$ samples), $451.2 \mu \mathrm{l}$ NFW to each tube (4.7 $\mu \mathrm{l}$ x 96 samples). 
- Add $412.8 \mu$ l of CPP1 HT to PCR1 Mastermix tube (4.3 $\mu$ x $x 96$ samples).

- Add $412.8 \mu$ of CPP2 HT to PCR2 Mastermix tube (4.3 $\mu \mathrm{l} \times 96$ samples).

c. Add $5 \mu$ l each of first strand cDNA from the cDNA plate to corresponding wells of COV1 and COV2 plates.

d. Add $20 \mu \mathrm{l}$ of PCR1 Mastermix to each well of COV1 plate.

e. Add $20 \mu \mathrm{l}$ of PCR2 Mastermix to each well of COV2 plate.

f. Seal and shake the COV1 and COV2 plates at 1600 rpm for 1 minute.

g. Centrifuge the plates at $1000 \times \mathrm{g}$ for 1 minute.

h. Place the plates on individual thermal cyclers and run the following program

- Choose the preheat lid option

- Set the final volume of the reaction to be $25 \mu \mathrm{l}$

- $\quad 98^{\circ} \mathrm{C}$ for 3 minutes

- 35 cycles of

- $\quad 98^{\circ} \mathrm{C}$ for 15 seconds

- $65^{\circ} \mathrm{C}$ for 5 minutes

- Hold at $4^{\circ} \mathrm{C}$.

i. The program has a run time of approximately 03 hours and 30 minutes.

Pause Point: The procedure can be paused here and the COV 1 and COV 2 plates can be stored at $-20^{\circ} \mathrm{C}$ for upto 3 days.

\section{Key Resources Table}

\begin{tabular}{|c|c|c|c|}
\hline Product Name & Catalog No. & Manufacturer & Links \\
\hline $\begin{array}{l}\text { Illumina COVIDSeq } \\
\text { Test } 3072 \text { samples }\end{array}$ & 20043675 & Illumina & $\begin{array}{l}\text { https://sapac.illumina.com/products/by- } \\
\text { type/ivd-products/covidseq.html }\end{array}$ \\
\hline $\begin{array}{l}\text { IDT for Illumina PCR } \\
\text { indexes Set 1-4 }\end{array}$ & 20043137 & Illumina & $\begin{array}{l}\text { https://emea.support.illumina.com/conte } \\
\text { nt/dam/illumina- } \\
\text { support/documents/documentation/che } \\
\text { mistry documentation/Illumina- } \\
\text { COVIDSeq-Test/Illumina-COVIDSeq-Test- } \\
\text { Reference-Guide-1000000126053-02.pdf }\end{array}$ \\
\hline $\begin{array}{l}\text { MiSeq reagent kit V3 } \\
\text { (150 cycles) }\end{array}$ & MS-102-3001 & Illumina & $\begin{array}{l}\text { https://sapac.illumina.com/products/by- } \\
\text { type/sequencing-kits/cluster-gen- } \\
\text { sequencing-reagents/miseq-reagent-kit- } \\
\text { v3.html }\end{array}$ \\
\hline $\begin{array}{l}\text { Qubit dsDNA HS } \\
\text { Assay kit }\end{array}$ & Q32851 & Thermofisher & $\begin{array}{l}\text { https://www.thermofisher.com/order/cat } \\
\text { alog/product/Q32851\#/Q32851 }\end{array}$ \\
\hline
\end{tabular}




\section{Materials and Equipment}

\begin{tabular}{|l|c|c|}
\hline Reagent & Storage & Total Volume Required per reaction \\
\hline SARS-CoV-2 RNA & $-20^{\circ} \mathrm{C}$ & $8.5 \mu \mathrm{l}$ \\
\hline CPC HT & $-20^{\circ} \mathrm{C}$ & $8.5 \mu \mathrm{l}$ \\
\hline EPH3 HT & $-20^{\circ} \mathrm{C}$ & $8.5 \mu \mathrm{l}$ \\
\hline FSM HT & $-20^{\circ} \mathrm{C}$ & $9 \mu \mathrm{l}$ \\
\hline RVT HT & $-20^{\circ} \mathrm{C}$ & $1 \mu \mathrm{l}$ \\
\hline IPM HT & $-20^{\circ} \mathrm{C}$ & $15 \mu \mathrm{l}$ \\
\hline CPP1 HT & $-20^{\circ} \mathrm{C}$ & $4.3 \mu \mathrm{l}$ \\
\hline CPP2 HT & $-20^{\circ} \mathrm{C}$ & $4.3 \mu \mathrm{l}$ \\
\hline TB1 HT & $4^{\circ} \mathrm{C}$ & $12 \mu \mathrm{l}$ \\
\hline EBLTS HT & $-20^{\circ} \mathrm{C}$ & $4 \mu \mathrm{l}$ \\
\hline ST2 HT & $4{ }^{\circ} \mathrm{C}$ & $10 \mu \mathrm{l}$ \\
\hline TWB HT & $4{ }^{\circ} \mathrm{C}$ & $200 \mu \mathrm{l}$ \\
\hline EPM HT & $-20^{\circ} \mathrm{C}$ & $24 \mu \mathrm{l}$ \\
\hline Index adapter & $-20^{\circ} \mathrm{C}$ & $10 \mu \mathrm{l}$ \\
\hline ITB & $\mathrm{RT}$ & $2 \mathrm{ml}$ \\
\hline $80 \%$ Ethanol & $\mathrm{RT}$ & $55 \mu \mathrm{l}$ \\
\hline RSB & $4{ }^{\circ} \mathrm{C}$ & $396 \mu \mathrm{l}$ (for 96 samples) \\
\hline
\end{tabular}

\section{Step-by-Step Method Details}

\section{Library Preparation and Library Pooling}

\section{Timing: [3-4 Hours]}

[In this step the prepared cDNA of the SARS-CoV-2 genome is tagmented, indexed and amplified to become sequencing-ready libraries.]

1. Tagmentation of PCR amplicons

a. Label a new 96-well PCR plate as TAG plate.

b. From COV1 and COV2 plates,combine $10 \mu$ l of contents from each well to their respective wells in TAG1 plate, and mix well. This results in a total volume of $20 \mu \mathrm{l}$ in each well of TAG1 plate.

c. To make Tagementation Mastermix:

- Label a $15 \mathrm{ml}$ tube as Tagmentation Mastermix.

- Add $1920 \mu \mathrm{l}$ of Nuclease-free water (20 $\mu \mathrm{l}$ x 96 samples).

- Add $1152 \mu$ l of TB1 HT (12 $\mu$ l x 96 samples).

- Add $384 \mu$ l of EBLTS HT (4 $\mu$ l x 96 samples).

- Vortex for 30 seconds to mix well.

d. Add $30 \mu \mathrm{l}$ of Tagmentation Mastermix to each well in the TAG1 plate.

e. Seal and shake the TAG1 plate at $1600 \mathrm{rpm}$ for 1 minute.

f. Centrifuge at $1000 \times \mathrm{g}$ for 1 minute if the contents of the well are found sticking to the walls or the seal of the TAG1 plate.

g. Place on a thermal cycler and run the following program. 
- Choose the preheat lid option

- Set the total volume of the reaction to be $50 \mu$ l.

- $55^{\circ} \mathrm{C}$ for 5 minutes

- Hold at $10^{\circ} \mathrm{C}$.

\section{Post Tagmentation Clean-up}

a. Centrifuge the TAG1 plate at $500 \times \mathrm{g}$ for 1 minute.

b. Remove the seal and add $10 \mu \mathrm{l}$ of ST2 HT to each well of the TAG1 plate.

c. Seal and shake at 1600 rpm for 1 minute.

d. Incubate the plate at room temperature for 5 minutes.

e. Centrifuge the TAG1 plate at $500 \times \mathrm{g}$ for 1 minute.

f. Remove the seal carefully and place the plate on a magnetic stand.

g. Wait for 3 minutes/until the liquid is clear.

h. Remove and discard all supernatant from the wells.

i. Wash the beads as follows

- Remove the plate from the magnetic stand.

- Add $100 \mu$ l of TWB HT to each well.

- Seal and shake at 1600 rpm for 1 minute and centrifuge at 500 x g for 1 minute.

- Remove the seal and place on the magnetic stand and wait for 3 minutes/until the liquid is clear.

- Remove and discard the supernatant from each well.

j. Repeat the wash steps (step i) one more time.

CRITICAL: Do not discard the supernatant after the second wash, to prevent the beads from over drying.

3. Amplification of Tagmented amplicons

a. In a $15 \mathrm{ml}$ tube, prepare Enhanced PCR Mix

- Add $2304 \mu \mathrm{l}$ of EPM HT (24 $\mu \mathrm{l} \times 96$ samples).

- Add $2304 \mu \mathrm{l}$ of Nuclease-free water ( $24 \mu \mathrm{l}$ x 96 samples).

- Vortex the tube to mix.

b. Remove and discard the supernatant from the TAG1 plate.

c. Use a $10 \mu \mathrm{l}$ or $20 \mu \mathrm{l}$ pipette to remove any remaining TWB HT from TAG1 plate.

d. Add $40 \mu \mathrm{l}$ of Enhanced PCR Mix to each well of the TAG1 plate.

e. Add $10 \mu \mathrm{l}$ of index adapters to each well of the TAG1 plate.

f. Seal and shake the plate at $1600 \mathrm{rpm}$ for 1 minute and centrifuge the plate at $500 \times \mathrm{g}$ for 1 minute.

CRITICAL: Inspect the tubes to make sure the beads are fully re-suspended and are not found at the bottom of the tubes.

g. Place on a thermal cycler and run the following PCR program

- Choose the preheat lid option and set the temperature to $100^{\circ} \mathrm{C}$.

- Set the total volume of the reaction to be $50 \mu$ l.

- $72^{\circ} \mathrm{C}$ for 3 minutes

- $98^{\circ} \mathrm{C}$ for 3 minutes

- 7 cycles of:

- $98^{\circ} \mathrm{C}$ for 20 seconds

- $60^{\circ} \mathrm{C}$ for 30 seconds

- $72^{\circ} \mathrm{C}$ for 1 minute

- $\quad 72^{\circ} \mathrm{C}$ for 3 minutes 
4. Library pooling and Clean up

a. Centrifuge the TAG1 plate at $500 \times \mathrm{g}$ for 1 minute.

b. Remove the seal and place the plate on a magnetic stand and wait for 3 minutes/until the liquid is clear.

c. Transfer $5 \mu \mathrm{l}$ of library from each well into an 8-tubes strip. This results in a pooled volume of $60 \mu \mathrm{l}$ in each tube of the 8-tubes strip.

CRITICAL: Discard and change tips for transferring each column of samples.

d. Transfer $55 \mu$ l of pooled library from each tube to a $1.5 \mathrm{ml}$ micro-centrifuge tube. This results in $440 \mu$ l of total pooled libraries (from 96 samples).

e. Add $396 \mu \mathrm{l}$ of ITB to each tube (ITB volume is calculated by multiplying the total volume of the pooled libraries with 0.9 i.e., for 96 samples: $440 \mu \mathrm{l} \times 0.9=396 \mu \mathrm{l}$ )

f. Vortex the tube to mix well.

g. Incubate the tube at room temperature for 5 minutes.

h. Spin briefly and place the tube on a $1.5 \mathrm{ml}$ tube magnetic stand and wait for 5 minutes.

i. Remove and discard all supernatant.

j. Wash the beads as follows:

- Add $1000 \mu$ l of freshly prepared $80 \%$ ethanol to the tube and wait for 30 seconds.

- Remove and discard the supernatant.

- Repeat the above mentioned wash steps one more time for a total of $\mathbf{2}$ washes with $80 \%$ ethanol.

k. Remove any residual ethanol left on the tube.

I. Air-dry the beads for 2 minutes.

m. Add $55 \mu$ l of Re-suspension Buffer to the tube and vortex to mix.

n. Incubate at room temperature for 2 minutes.

o. Spin briefly and place the tube in the magnetic stand and wait for 2 minutes.

p. Label a new $1.5 \mathrm{ml}$ tube as Final Pool.

q. Transfer $50 \mu$ l of the supernatant into the Final Pool tube.

Pause Point: The procedure can be paused here and the Final Pool tube can be stored at $-20^{\circ} \mathrm{C}$ for 30 days.

5. Quantification and Normalization of the library pool

a. Using the Qubit High Sensitivity dsDNA quantification kit (Invitrogen), quantify the library pool

- Dilute $1 \mu$ l of the pooled library sample in the ratio of 1:3.

- Use $2 \mu$ l of the diluted library for quantification.

- Calculate the actual concentration of the library by factoring in the dilution.

b. Load $2 \mu$ l of the pooled library (undiluted) onto a $2 \%$ agarose gel. The libraries should be observed between the size 300-400 bp.

c. Using the formula below, calculate the concentration of the library in Molar units.

d. Dilute the library with Resuspension Buffer to a final concentration of $4 \mathrm{nM}$.

e. Formula for calculating Molarity:

[Library Conc $(\mathrm{ng} / \mu \mathrm{l})] /[660(\mathrm{~g} / \mathrm{mol}) \times$ average library size $(\mathrm{bp})] \times 10^{6}=$ Conc. of Library $(\mathrm{nM})$ 


\section{Sequencing the Libraries}

\section{Timing: [ 22 hours: according to MiSeq reagent kit V3 (2 x 75bp)]}

[The prepared COVIDSeq libraries will be sequenced in this step using the MiSeq sequencing platform]

6. Preparing PhiX

a. Label a new $1.5 \mathrm{ml}$ tube as PhiX Control.

b. Combine $2 \mu$ l of PhiX stock library (10nM) and $3 \mu$ l of Nuclease-free water to make 4nM PhiX library

CRITICAL: Prepare a fresh PhiX dilution and use it within 12 hours of preparation.

c. Label a new $1.5 \mathrm{ml}$ tube as PhiX-Final and add $5 \mu \mathrm{l}$ of diluted PhiX (4nM) and $5 \mu \mathrm{l}$ of $0.2 \mathrm{~N}$ freshly prepared $\mathrm{NaOH}$.

d. Vortex the tube briefly to mix. Spin and incubate the tube at room temperature for 5 minutes.

e. Add $990 \mu$ l of pre-chilled HT1 to the PhiX-Final tube resulting in $1 \mathrm{ml}$ of $20 \mathrm{pM}$ PhiX library.

2. Denaturation of COVIDSeq library

a. Label a new $1.5 \mathrm{ml}$ tube as Library-Final and add $5 \mu \mathrm{l}$ of diluted library (4nM) and $5 \mu \mathrm{l}$ of $0.2 \mathrm{~N}$ freshly prepared $\mathrm{NaOH}$.

b. Vortex the tube briefly to mix. Spin and incubate the tube at room temperature for 5 minutes.

c. Add $990 \mu \mathrm{l}$ of pre-chilled HT1 to the tube resulting in $1 \mathrm{ml}$ of $20 \mathrm{pM}$ Final library.

CRITICAL: For instructions regarding final loading concentration and PhiX spike in for MiSeq system, refer to the MiSeq system Denature and Dilute Libraries Guide.

d. To prepare $6 \mathrm{pM}$ final loading concentration library

- Label a new $1.5 \mathrm{ml}$ tube as 6pM Final Library tube.

- Add $180 \mu$ l of the $20 p M$ library from Library-Final tube.

- Add $420 \mu$ l of prechilled HT1 and mix well.

- Discard $6 \mu$ l of the mixture.

- Add $6 \mu$ l of the 20pM PhiX library for $1 \%$ spike in as recommended by Illumina Inc.

- Mix well with a pipette, spin briefly and place the tube on ice. The library is ready to be loaded onto the flow cell.

CRITICAL: The final library has to be loaded onto the flow cell within 30 minutes of preparation.

NOTE: To reduce the handling time, the denaturation of library and PhiX can be performed simultaneously.

3. Sequencing and expected Output

a. Load the library into the MiSeq Sequencing cartridge and initiate sequencing as per the MiSeq Sequencing guide by Illumina Inc.

b. For sequencing using MiSeq v3 $2 \times 75$ cycles kit, the estimated yield is about 3.3-3.8 Gb as per Illumina Inc. 


\section{Expected Outcomes}

Upon successful tagmentation and the size selection the final library pool will appear as $300 \mathrm{bp}$ size band on $2 \%$ agarose gel. Any deviation in the fragment size may be the result of erroneous tagmentation or the size selection, which can me minimise with proper handling and pipetting practices.

\section{Quantification and Statistical Analysis}

\section{Genome Assembly, Variant Calling and Phylogenetic Analysis}

This step describes the basic bioinformatics pipeline for genome assembly and identification of variants in the SARS-CoV-2 genome. The raw sequencing data generated by Illumina sequencing platforms are in the form of Binary Base Call (BCL) files and require conversion to FASTQ format before further processing.

1. The BCL files generated by the sequencer are demultiplexed to FASTQ files using the bcl2fastq conversion software provided by Illumina following the command:

bcl2fastq --runfolder-dir <path to the directory of the sequencing run files> --sample-sheet <path to the sample sheet>--output-dir <path to demultiplexed output folder>

2. The raw FASTQ files need to undergo additional processing steps, including quality control, trimming, alignment against the SARS-CoV-2 reference genome and sorting. The resulting BAM file containing mapped and sorted reads are then used to call variants and generate a consensus sequence of the genomes in FASTA file format. The steps to assemble SARS-CoV-2 genomes from raw FASTQ files are detailed in a recent protocol (Poojary et al 2020). Sample parameters and metrics that may be used to assess the quality of the assembled genomes are given in Table 1.

3. For assignment of lineages to the assembled genome FASTA sequences, we use the Phylogenetic Assignment of Named Global Outbreak Lineages (PANGOLIN) software command line tool using the command:

pangolin <FASTA sequence file name $>$. fasta

Alternatively, the web version of PANGOLIN (available at https://pangolin.cog-uk.io/) can be used to assign lineages to the generated FASTA sequences (Rambaut et al 2020).

4. The FASTA sequence files can also be used to analyse and visualise evolutionary relationships through phylogenetic clustering. For performing the phylogenetic analysis, we use the opensource tool-kit Nextstrain following a recently detailed protocol (Jolly and Scaria 2021) 
Table 1

\begin{tabular}{|l|l|l|l|l|l|l|l|l|l|l|l|}
\hline $\begin{array}{l}\text { Sampl } \\
\text { e ID }\end{array}$ & $\begin{array}{l}\text { Numb } \\
\text { er of } \\
\text { Raw } \\
\text { Reads } \\
\text { R1 }\end{array}$ & $\begin{array}{l}\text { Numb } \\
\text { er of } \\
\text { Raw } \\
\text { Reads } \\
\text { R2 }\end{array}$ & $\begin{array}{l}\text { Numb } \\
\text { er of } \\
\text { Reads } \\
\text { after } \\
\text { trimmi } \\
\text { ng R1 }\end{array}$ & $\begin{array}{l}\text { Numb } \\
\text { er of } \\
\text { Reads } \\
\text { after } \\
\text { trimm } \\
\text { ing R2 }\end{array}$ & $\begin{array}{l}\text { \% of } \\
\text { Reads } \\
\text { Aligne } \\
\text { d with } \\
\text { Refere } \\
\text { nce }\end{array}$ & $\begin{array}{l}\text { Numbe } \\
\text { r of } \\
\text { Base } \\
\text { Pairs } \\
\text { Covere } \\
\text { d }\end{array}$ & $\begin{array}{l}\text { X } \\
\text { cover } \\
\text { age }\end{array}$ & $\begin{array}{l}\text { Geno } \\
\text { me } \\
\text { Cover } \\
\text { age }\end{array}$ & $\begin{array}{l}\text { Numb } \\
\text { er of } \\
\text { varian } \\
\text { ts } \\
\text { Called }\end{array}$ & $\begin{array}{l}\text { PANG } \\
\text { O } \\
\text { Linea } \\
\text { ge }\end{array}$ & $\begin{array}{l}\text { Time } \\
\text { Taken }\end{array}$ \\
\hline $\begin{array}{l}\text { CSXXX } \\
\text { X }\end{array}$ & $\begin{array}{l}14319 \\
19\end{array}$ & $\begin{array}{l}14319 \\
19\end{array}$ & $\begin{array}{l}12215 \\
80\end{array}$ & $\begin{array}{l}12215 \\
80\end{array}$ & $99.52 \%$ & $\begin{array}{l}843103 \\
80\end{array}$ & $\begin{array}{l}2819 . \\
46\end{array}$ & $\begin{array}{l}99.84 \\
\%\end{array}$ & 11 & B.1.36 & $\begin{array}{l}318 \\
\text { secon } \\
\text { ds }\end{array}$ \\
\hline
\end{tabular}

\section{Limitations}

As explained in our previous report (Bhoyar et al 2021) the Illumina COVIDSeq can detect the SARSCoV-2 RNA ranging from the highest $C_{T} 37$ to the lowermost $C_{T}$ sample. However, we have noticed that in order to capture the whole SARS-CoV-2 genome, RNA samples below $C_{\mathrm{T}} 22$ should consider.

\section{Troubleshooting}

Problem 1:

Amplification in the negative control (NC)

In some cases, users may get the amplification in the negative control well in the COVIDSeq assay.

Potential Solution:

To avoid the amplification in the negative control we encourage the users to stick to the following practices.

- Autoclave all the pipettes before initiating the new experiment.

- Thoroughly decontaminate the Biosafety cabinet before the experiment.

- Use fresh PCR plate sealing films every time.

\section{Resource Availability Lead Contact}

Further information and requests for resources and reagents should be directed to and will be fulfilled by the Lead Contact, Dr. Sridhar Sivasubbu (sridhar@igib.in).

\section{Materials Availability}

All the materials used in the protocol are available without any restrictions; the details of the kits used in the protocol are given in the "key resource table". 


\section{Data and Code Availability}

All the scripts used for the SARS-CoV-2 sequencing analysis and interpretation are adopted from Poojary et al 2020.

\section{Acknowledgments}

Authors acknowledge funding from the Council of Scientific and Industrial Research (CSIR), India through grants CODEST and MLP2005. The funders had no role in the design of experiment, analysis or decision to publish.

\section{Author Contributions}

All of the authors contributed substantially to the conception and design of the study, the acquisition of data, analysis and interpretation. S.S.B. and V.S. Funding Acquisition.

\section{Declaration of Interests}

The authors declare no competing interests.

\section{References}

Bhoyar, R.C., Jain, A., Sehgal, P., Divakar, M.K., Sharma, D., Imran, M., Jolly, B., Ranjan, G., Rophina, M., Sharma, S., et al. (2021). High throughput detection and genetic epidemiology of SARS-CoV-2 using COVIDSeq next-generation sequencing. PLoS One 16, e0247115.

Poojary, M., Shantaraman, A., Jolly, B., and Scaria, V. (2020). Computational protocol for assembly and analysis of SARS-nCoV-2 genomes. Research Reports 4: e1-e14 doi:10.9777/rr.2020.10001

Rambaut, A., Holmes, E.C., O’Toole, Á. et al. (2020). A dynamic nomenclature proposal for SARS-CoV-2 lineages to assist genomic epidemiology. Nat Microbiol 5, 1403-1407.

https://doi.org/10.1038/s41564-020-0770-5

Hadfield J., Megill C., Bell SM., Huddleston J., Potter B.,et al. (2018). Nextstrain: real-time tracking of pathogen evolution. Bioinformatics.1;34(23):4121-4123. doi: 10.1093/bioinformatics/bty407.

Jolly, B. and Scaria, V. (2021). Computational Analysis and Phylogenetic Clustering of SARS-CoV-2 Genomes. Bio-protocol 11(8): e3999. doi: 10.21769/BioProtoc.3999. 